04

\title{
Влияние побочных дифракционных порядков на качество изображения, формируемого рефракционно-дифракционной оптической системой среднего ИК диапазона
}

\author{
(С) Г.И. Грейсух, Е.Г. Ежов, О.А. Захаров, С.В. Казин \\ Пензенский государственный университет архитектуры и строительства, \\ 440028 Пенза, Россия \\ e-mail: grey@pguas.ru
}

Поступила в редакцию 16.12.2020 г.

В окончательной редакции 16.12.2020 г.

Принята к публикации 22.12.2020 г.

\begin{abstract}
Путем компьютерного моделирования и экспериментально исследовано влияние побочных дифракционных порядков дифракционного корректора хроматизма на качество изображения вблизи оптической оси, формируемого рефракционно-дифракционной оптической системой, рассчитанной на излучение среднего ИК диапазона $(3-5 \mu \mathrm{m})$. Показано, что побочные дифракционные порядки однослойной пилообразной рельефнофазовой микроструктуры, дифракционная эффективность которой на центральной длине волны рабочего спектрального диапазона близка к единице, не будут ощутимо влиять на качество изображения при условии, что дифракционная эффективность в пределах всего указанного спектрального диапазона не опускается ниже 0.67 .
\end{abstract}

Ключевые слова: ИК гибридная германиевая асферическая линза, дифракционный корректор хроматизма, рельефно-фазовая микроструктура, дифракционная эффективность, побочные дифракционные порядки.

DOI: $10.21883 /$ OS.2021.04.50763.273-20

\section{Введение}

Возможности совершенствования рефракционных оптических систем во многом связаны с расширением той области на диаграмме Аббе, которую занимают доступные оптические материалы. И если область на этой диаграмме, занимаемая технологичными материалами, прозрачными в видимом спектральном диапазоне, сегодня расширяется достаточно быстро, то в ИК диапазоне ситуация значительно скромнее. Ассортимент кроно- и флинтоподобных технологичных материалов, прозрачных в среднем $(3 \leq \lambda \leq 5 \mu \mathrm{m})$ или дальнем $(8 \leq \lambda \leq 12 \mu \mathrm{m})$ ИК диапазонах, весьма ограничен. Поэтому уникальные аберрационные свойства дифракционных оптических элементов наиболее важное значение имеют именно для ИК диапазона.

Действительно, введение дифракционного корректора (ДК) в рефракционный объектив тепловизора позволяет упростить его оптическую схему и достичь при этом требуемых оптических характеристик (см., например, $[1,2]$ ). Так, в частности, в среднем ИК диапазоне размещение дифракционной микроструктуры на плоской поверхности одной из рефракционных линз высокоапертурного триплета позволило одновременно выполнить условия коррекции как хроматических, так и монохроматических аберраций и при этом отказаться от использования в триплете германия, обладающего высоким и нелинейным температурным коэффициентом показателя преломления. Благодаря дифракционной микроструктуре при замене германия на халькогенидное стекло была сохранена апохроматизация и обеспечен низкий уровень сферохроматизма $[3,4]$.

Однако дифракционный механизм преобразования волнового фронта на микроструктуре ДК приводит к тому, что в рефракционно-дифракционных оптических системах на изображение, сформированное излучением, дифрагированным в рабочий порядок дифракции, накладывается паразитное излучение, сформированное за счет дифракции на этой же микроструктуре в побочные дифракционные порядки.

Влияние побочных дифракционных порядков на качество изображения, формируемого рефракционнодифракционной оптической системой видимого спектрального диапазона, достаточно хорошо исследовано (см., например, [5-9]). В то же время публикации результатов аналогичных исследований для ИК диапазона отсутствуют. Настоящая работа призвана заполнить указанный пробел.

\section{Гибридная германиевая линза и ее дифракционная эффективность}

Общность результатов исследования влияния побочных дифракционных порядков на качество изображения будет во многом определяться оптической схемой исследуемого рефракционно-дифракционного объектива. При работе ДК в первом порядке дифракции эта схема, как минимум, должна обеспечить высокое качество полихроматического изображения вблизи оптической оси. Для 


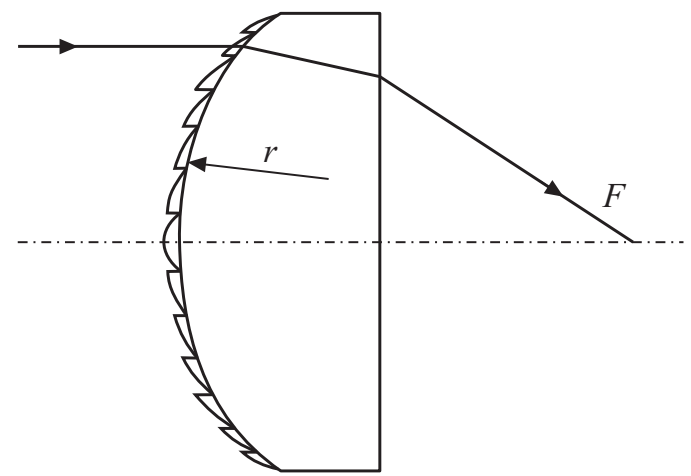

Рис. 1. Оптическая схема Hybrid Ge Aspheric Lens No 68260: радиус кривизны при вершине асферической поверхности $r=46.2 \mathrm{~mm}$; осевая толщина $d=4 \mathrm{~mm}$; световой диаметр $D=25 \mathrm{~mm}$.

этого уровень ее сферической аберрации, хроматизма положения и сферохроматизма должен быть достаточно низким. Очевидно, что в этом случае работа корректора в побочных порядках будет приводить к значительному снижению качества изображения.

Вышеприведенные условия нетрудно выполнить, используя в качестве исследуемого объектива, например, гибридную германиевую асферическую линзу (ГГАЛ) Hybrid Ge Aspheric Lens No 68-260, серийно производимую фирмой Edmund Optics [10]. Оптическая схема ГГАЛ представлена на рис. 1 .

ДК, т.е. кольцевая дифракционная микроструктуpa, выполненная на асферической поверхности, имеет небольшую оптическую силу и обеспечивает ахроматическую коррекцию ГГАЛ в рабочем спектральном диапазоне $(3 \leq \lambda \leq 5 \mu \mathrm{m})$. Эффективное фокусное расстояние ГГАЛ на центральной длине волны $(\bar{\lambda}=4 \mu \mathrm{m})$ рабочего спектрального диапазона $f^{\prime \prime} \mathrm{HGAL}=15 \mathrm{~mm}$, а фокусное расстояние ДК в рабочем +1-м порядке дифракции $f^{\prime \prime} \mathrm{DC}=845.24 \mathrm{~mm}$.

Сферическая аберрация ГГАЛ для $D=25 \mathrm{~mm}$ полностью устранена. Она не превышает $0.005 \lambda$ на длине волны $\lambda=\bar{\lambda}$ для осевого точечного источника, удаленного в бесконечность со стороны ДК. В то же время для снижения сферохроматизма до приемлемого уровня необходимо ограничить световой диаметр ГГАЛ до $D_{w}=11 \mathrm{~mm}$.

При $D=25 \mathrm{~mm}$ микроструктура ГГАЛ включает 20 зон Френеля, а при $D_{w}=11 \mathrm{~mm}$ - только 4 зоны. Здесь напомним, что применительно к пилообразной рельефно-фазовой микроструктуре под термином „зона Френеля“" понимается участок микроструктуры, в пределах которого фазовая задержка, вносимая микроструктурой в падающий на нее волновой фронт, изменяется от 0 до $2 \pi$ [11-13].

Глубина пилообразного рельефа микроструктуры ГГАЛ выбрана из условия обеспечения максимальной дифракционной эффективности (ДЭ), близкой к 100\%, на $\bar{\lambda}$ в +1 -м рабочем порядке дифракции. Она в соответствии с формулой $[12,13]$

$$
h=\bar{\lambda} /(\bar{n}-1)
$$

составляет $h=1.32 \mu \mathrm{m}$, так как показатель преломления материала GERMANIUM, указанного в Zemaxфайле [14], прилагаемом производителем к ГГАЛ, на длине волны $\bar{\lambda}$ равен $\bar{n}=4.025058$. Просветляющие покрытия обеих рабочих поверхностей ГГАЛ обеспечивают коэффициент отражения $R_{\text {avg }}<3 \%$ во всем рабочем спектральном диапазоне.

При вышеуказанном числе зон Френеля и при минимальном значения нормированного пространственного периода микроструктуры $\Lambda_{\min } / h>530$ реальная ДЭ, которая может быть оценена путем численного решения уравнений Максвелла, практически совпадает с оценкой, получаемой в рамках скалярной теории дифракции и в приближении бесконечно тонкого транспаранта $[5,7]$. Поэтому ниже для оценки ДЭ в различных дифракционных порядках будем использовать выражение, полученное в скалярном приближении и приведенное, например, B [5]:

$$
\eta=\left\{\frac{\sin [\pi(\Delta l / \lambda-m)]}{\pi(\Delta l / \lambda-m)}\right\}^{2}
$$

где $\lambda$ - длина волны падающего на микроструктуру света, $\Delta l-$ приращение оптического пути на одном периоде пилообразного профиля, и в нашем случае $\Delta l=(n-1) h$, a $n-$ показатель преломления материала микроструктуры на длине волны $\lambda$. Распределение ДЭ по порядкам дифракции на ряде длин волн, на которых ДЭ > 0.001, представлено в табл. 1.

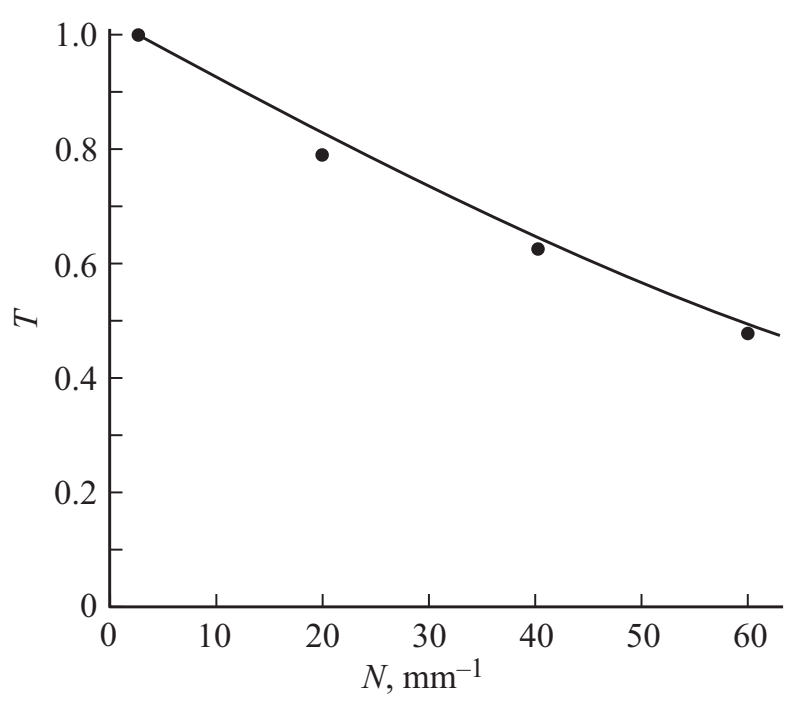

Рис. 2. ЧКХ ГГАЛ, рассчитанная в ПНУ для бесконечно удаленной осевой предметной точки: без учета (сплошная линия) и с учетом влияния побочных дифракционных порядков. 
Таблица 1. Распределение ДЭ микроструктуры ГГАЛ по порядкам дифракции

\begin{tabular}{c|c|c|c|c|c|c|c|c}
\hline \multirow{2}{*}{$\lambda, \mu \mathrm{m}$} & \multicolumn{9}{|c}{ Номер порядка дифракции } \\
\cline { 2 - 10 } & -2 & -1 & 0 & +1 & +2 & +3 & +4 & +5 \\
\hline 3 & 0.0070 & 0.0143 & 0.0435 & 0.6700 & 0.1808 & 0.0285 & 0.0111 & 0.0059 \\
\hline 3.5 & 0.0020 & 0.0043 & 0.0152 & 0.9315 & 0.0274 & 0.0058 & 0.0024 & 0.0013 \\
\hline 4 & 0 & 0 & 0 & 1 & 0 & 0 & 0 & 0 \\
\hline 4.5 & 0.0015 & 0.0035 & 0.0159 & 0.9579 & 0.0010 & 0.0028 & 0.0013 & 0.0007 \\
\hline 5 & 0.0046 & 0.0111 & 0.0561 & 0.8724 & 0.0247 & 0.0074 & 0.0035 & 0.0020
\end{tabular}

\section{Результаты компьютерных исследований в предположении равномерности спектра ИК излучения}

Из-за небольших размеров излучающих элементов искусственных калиброванных источников ИК излучения исследование ГГАЛ с предметной плоскостью, удаленной в бесконечность, было ограничено компьютерным моделированием в программной среде Zemax [14]. При этом предполагалось, что энергетическая светимость точечного источника и миры одинаковы на всех длинах волн рабочего спектрального диапазона. Благодаря этому результаты исследования определялись исключительно свойствами оптической системы и не зависели от свойств источника излучения.

На рис. 2 (сплошная линия) представлена полихроматическая частотно-контрастная характеристика (ЧКХ) ГГАЛ, рассчитанная в плоскости наилучшей установки (ПНУ) $s^{\prime}=13.996 \mathrm{~mm}$ для бесконечно удаленной осевой предметной точки. Эта характеристика рассчитана для +1-го порядка дифракции по 5 длинам волн, представленным в табл. 1, с учетом ДЭ на каждой длине волны в виде весовых коэффициентов. Расчет выполнялся в среде Zemax в программе „Huygens MTF“ без учета влияния побочных порядков дифракции. Учитывая, что ни одна программа среды Zemax не позволяет непосредственно получить полихроматическую ЧКХ с учетом влияния побочных порядков дифракции, эта процедура выполнялась в два этапа. На первом этапе в программе „Huygens MTF“ с учетом побочных порядков дифракции рассчитывались монохроматические ЧКХ для 5 длин волн, представленных в табл. 1. На втором этапе контраст на выбранной пространственной частоте вычислялся с использованием известной формулы Д.Ю. Гальперна [15], которая для ряда длин волн рабочего спектрального диапазона приводилась к виду

$$
K_{p}(N)=\frac{\sum_{\lambda_{1}}^{\lambda_{I}} \tau_{\lambda} K_{\lambda}(n)}{\sum_{\lambda_{1}}^{\lambda_{I}} \tau_{\lambda}},
$$

где $\tau_{\lambda}-$ весовой коэффициент, равный в общем случае произведению относительной мощности спектральной линии источника излучения на пропускание и ДЭ на этой спектральной линии, $K_{\lambda}(N)-$ контраст на этой спектральной линии и на выбранной пространственной частоте $N$.

Контраст на пространственных частотах 20, 40 и $60 \mathrm{~mm}^{-1}$ в предположении равенства единице относительных мощностей всех спектральных линий источника излучения также показан на рис. 2.

Рисунок 2 показывает, что побочные порядки дифракции ощутимо влияют на ЧКХ только на низких пространственных частотах. Аналогичная ситуация наблюдается и в видимом диапазоне, где отрицательное влияние побочных порядков проявляется в основном в виде ореола (гало), окаймляющего контуры изображения. Цвет гало соответствует той части рабочего спектра излучения, на которой ДЭ дифракционного элемента оптической системы наименьшая [9].

Причину возможного образования гало в изображении, формируемом ГГАЛ, наглядно демонстрирует рис. 3. Действительно, сопоставляя сечения дифракционных изображений $(a)$ и $(b)$, легко видеть, что побочные дифракционные порядки приводят к образованию пьедестала, который и наблюдается в виде гало. Это подтверждается и эмуляцией изображения 4-шпальной миры, формируемой ГГАЛ в той же самой ПНУ на одной длине волны, равной $3 \mu \mathrm{m}$ (рис. 4). Размер изображения $2 \times 2 \mathrm{~mm}^{2}$. Здесь и далее если особо не оговорено, то, как принято на чертежах и иллюстрациях в оптике, элементы контрастного объекта черные на белом фоне, а в плоскости изображения уровень серого пропорционален энергетической освещенности, представленной шкалой справа от поля изображения.

Наблюдается гало (пусть и не так отчетливо) и в полихроматическом изображении (рис. 5).

Представленные выше результаты позволяют сделать вывод о том, что в среднем ИК диапазоне побочные дифракционные порядки дифракционного элемента с пилообразной однослойной микроструктурой существенного влияния на качество формируемого изображения оказывать не будут.

Обеспечить расчетный режим работы ГГАЛ с источником ИК излучения конечных размеров можно, разместив источник в фокальной плоскости ГГАЛ со стороны ее плоской поверхности. Для переноса же 

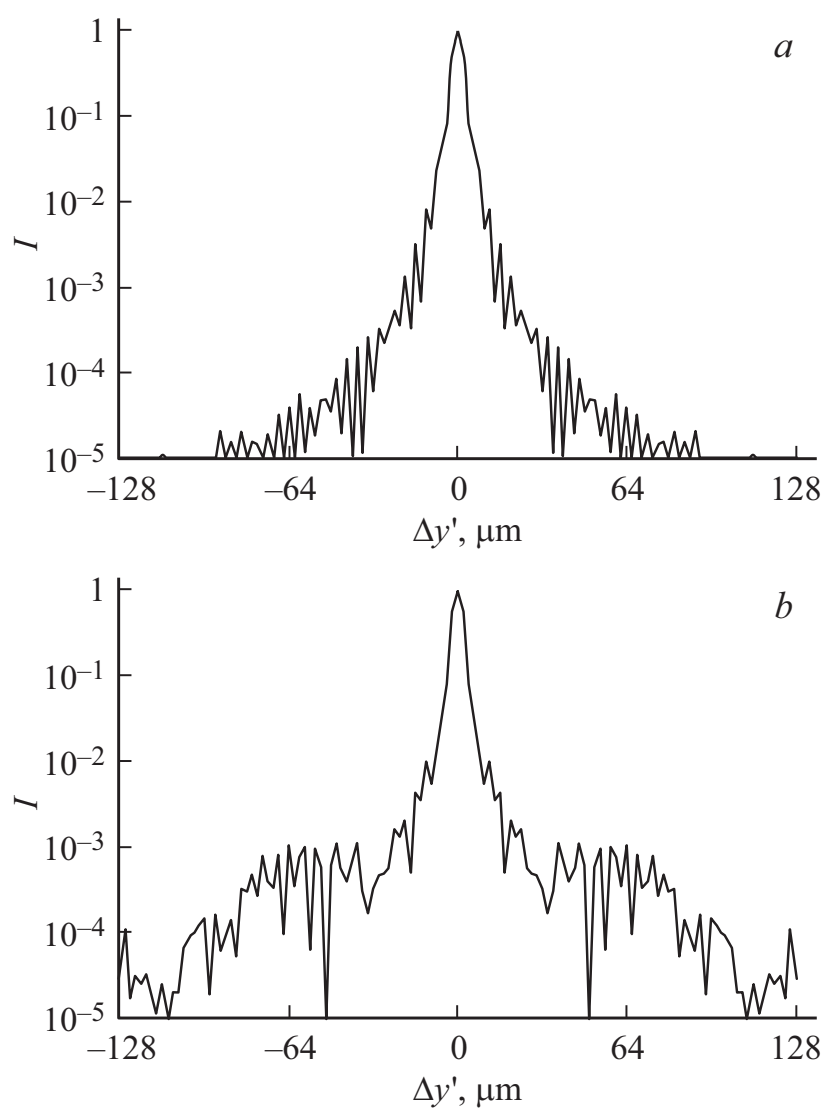

Рис. 3. Сечения дифракционных изображений в ПНУ бесконечно удаленного осевого точечного источника, сформированных ГГАЛ на длине волны $3 \mu \mathrm{m}: a-$ без учета влияния побочных дифракционных порядков, $b-$ с учетом влияния побочных дифракционных порядков.

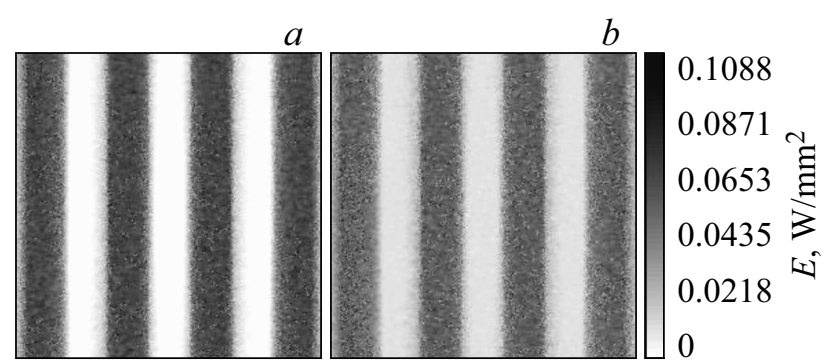

Рис. 4. Выполненная в программе „Image Simulation“ среды Zemax эмуляция изображения 4-шпальной миры, формируемой ГГАЛ в той же самой ПНУ на одной длине волны, равной $3 \mu \mathrm{m}$ : $a-$ без учета влияния побочных дифракционных порядков, $b-$ с учетом влияния побочных дифракционных порядков.

плоскости изображения из бесконечности на требуемое расстояние необходим дополнительный оптический компонент. В результате приходим к оптической схеме двухкомпонентной системы с параллельным ходом лучей между компонентами. В простейшем случае в качестве второго компонента может быть использована одиночная плосковыпуклая асферическая линза с фокусным расстоянием, близким к фокусному расстоянию ГГАЛ, с устраненной сферической аберрацией, выполненная из кроноподобного материала, прозрачного в среднем ИК диапазоне. При этом степень нарушения ахроматизации, т.е. разница задних отрезков оптической системы на крайних длинах волн рабочего спектрального диапазона будет тем меньше, тем больше коэффициент дисперсии оптического материала этой линзы.

Вышеперечисленным требованиям в достаточной степени удовлетворяет встроенная германиевая линза ИК видеокамеры HTPA80x64d [16]. На длине волны $\bar{\lambda}=4 \mu \mathrm{m}$ она имеет меньшее, чем у ГГАЛ, фокусное расстояние $\left(f^{\prime \prime} \mathrm{GL}=10.5 \mathrm{~mm}\right)$, но, как и требуется, при световом диаметре $11 \mathrm{~mm}$ свободна от сферической аберрации. Что же касается хроматизма положения и сферохроматизма двухкомпонентной оптической системы в целом, то они в 3 и 6 раз превосходят соответствующие аберрации ГГАЛ, достигая уровня, типичного для ИК оптики среднего ценового диапазона.

На рис. 6 и 7 представлены сечения дифракционных изображений осевого точечного источника и эмуляции изображения 4-шпальной миры, сформированные двухкомпонентной рефракционно-дифракционной оптической системой.

Сопоставляя рис. 6 и 3, нетрудно видеть, что в случае двухкомпонентной оптической системы образование пьедестала, обусловленного побочными дифракционными порядками, не столь ярко выражено из-за большего уровня хроматизма положения и сферохроматизма. Соответственно не столь ярко проявляется и гало, что легко видеть, сопоставляя рис. 7 и 4.

Таким образом, по результатам компьютерного анализа и моделирования можно сделать вывод, что гало, обусловленное падением на какой-то длине волны ДЭ пилообразной микроструктуры ДК рефракционнодифракционной оптической системы ИК диапазона не ниже 0.67 , не приведет к ощутимому снижению качества изображения. Этот вывод подтверждают и результаты экспериментальных исследований.

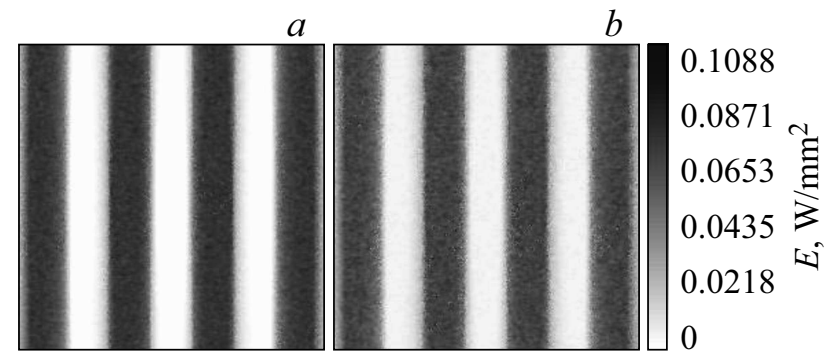

Рис. 5. Выполненная в программе „Image Simulation“ среды Zemax эмуляция изображения 4-шпальной миры, формируемой ГГАЛ в той же самой ПНУ на 5 длинах волн, представленных в табл. 1: $a-$ без учета влияния побочных дифракционных порядков, $b-\mathrm{c}$ учетом влияния побочных дифракционных порядков. 


\section{Результаты компьютерных и экспериментальных исследований с ИК источником теплового излучения}

В реальных условиях оптическая ИК система, как правило, работает с тепловым излучением, подобным
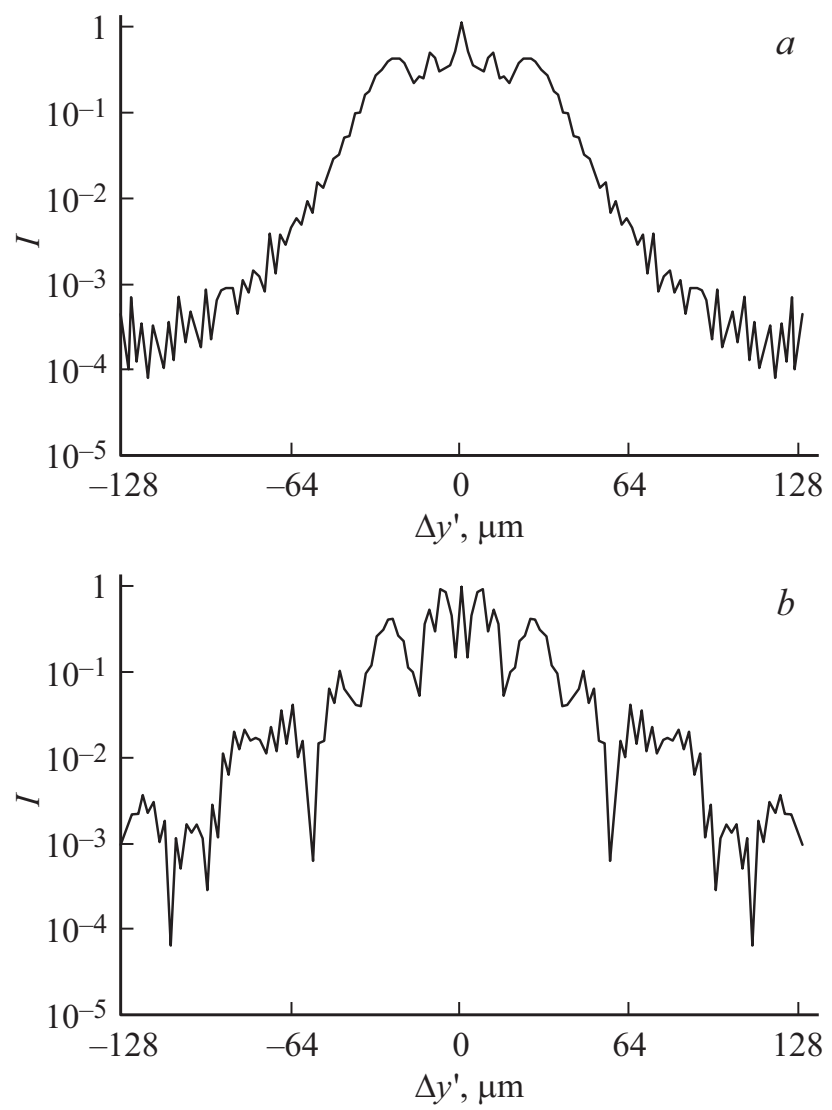

Рис. 6. Сечения дифракционных изображений в ПНУ $\left(s^{\prime}=9.506 \mathrm{~mm}\right)$ осевого точечного источника, сформированных двухкомпонентной рефракционно-дифракционной оптической системой на длине волны $3 \mu \mathrm{m}: a-$ без учета влияния побочных дифракционных порядков, $b-$ с учетом влияния побочных дифракционных порядков.

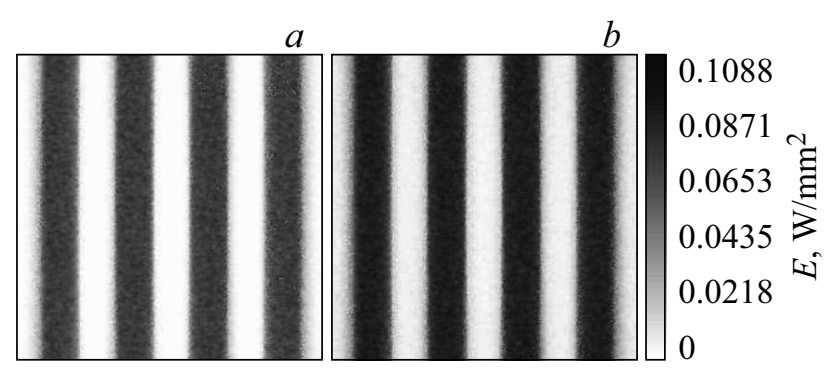

Pис. 7. Выполненная в программе „Image Simulation“ среды Zemax эмуляция изображения 4-шпальной миры, формируемой двухкомпонентной рефракционно-дифракционной оптической системой в той же самой ПНУ на 5 длинах волн, представленных в табл. 1: $a-$ без учета влияния побочных дифракционных порядков, $b-\mathrm{c}$ учетом влияния побочных дифракционных порядков.

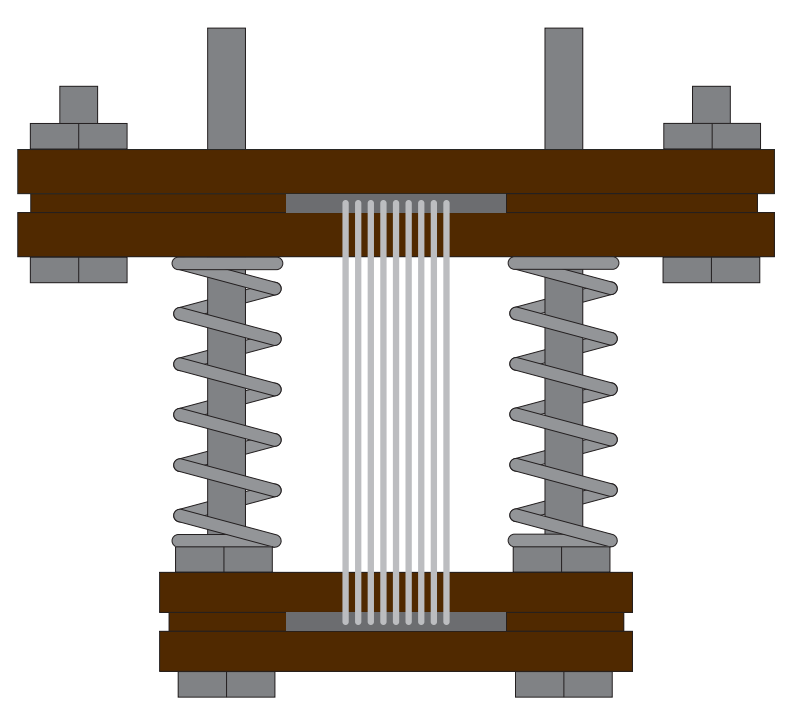

Рис. 8. Общий вид источника ИК излучения с плоской спиралью.

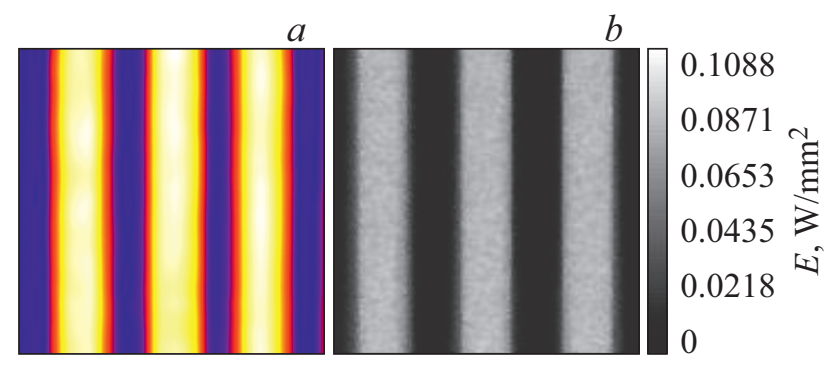

Рис. 9. Сформированное ГГАЛ совместно с ИК видеокамерой изображение нагревательного элемента $(a)$ и его эмуляция $(b)$.

Таблица 2. Нормированная спектральная плотность энергетической светимости излучателя при $T_{c}=725 \mathrm{~K}$ и ДЭ микроструктуры ГГАЛ в +1-м порядке дифракции на соответствующих длинах волн

\begin{tabular}{c|c|c|c|c|c|c|c}
\hline \multirow{2}{*}{ Параметр } & \multicolumn{7}{|c}{ Длина волны, $\mu \mathrm{m}$} \\
\cline { 2 - 8 } & 2 & 3 & 4 & 5 & 6 & 7 & 8 \\
\hline$M_{e \lambda} / M_{e, \lambda}^{(\max )}$ & 0.22 & 0.80 & 1 & 0.90 & 0.72 & 0.53 & 0.40 \\
\hline ДЭ & 0.0004 & 0.67 & 1 & 0.87 & 0.67 & 0.50 & 0.37
\end{tabular}

излучению абсолютно черного тела. Так, в частности, при цветовой температуре излучателя $T_{c}=725 \mathrm{~K}$ максимум его спектральной плотности энергетической светимости $M_{e . \lambda}^{(\max )}$, согласно формуле Планка, приходится на $\bar{\lambda}=4 \mu$ m и падает до уровня 0.5 на длинах волн 2.45 и $7.3 \mu \mathrm{m}$ (табл. 2).

В программы расчета и моделирования среды Zemax могут быть внесены значения $M_{e . \lambda}^{(\max )}$ в качестве весовых коэффициентов, что позволяет оценить качество формируемого оптической системой изображения с учетом 
свойств источника излучения. Авторы воспользовались этой возможностью для сопоставления экспериментальных результатов и результатов компьютерного моделирования.

Первоначально в качестве источника излучения среднего ИК диапазона в эксперименте предполагалось использовать серийно выпускаемый источник с отражателем EMIRS200_AT01T_BR090 [17]. Излучатель этого источника представляет собой прямоугольную площадку размером $2.1 \times 1.8 \mathrm{~mm}^{2}$. Однако из-за значительной неравномерности температуры, а следовательно, и светимости (температура краев площадки и особенно ее углов существенно меньше, чем центра) более информативным оказалось использование в качестве источника излучения изготовленного авторами нагревательного элемента в виде плоской спирали, выполненной из нихромовой проволоки диаметром $0.5 \mathrm{~mm}$ (рис. 8). Спираль содержала 9 прямолинейных участков (нитей), что обеспечивало равенство температур 3 центральных из них, которые вводились в поле зрения ГГАЛ и видеокамеры.

Цветовая температура измерялась мультиметром Vici VC99 3 6/7 DMM с термопарой TP-01 K-type Thermocouple Sensor. На рис. 9 представлены сформированное ГГАЛ совместно с ИК видеокамерой HТРА80x64d изображение нагревательного элемента и его эмуляция. Эмуляция, как и выше, выполнялась в программе „Image Simulation“ среды Zemax, а изображение выводилось на экран инвертированным (светлые элементы на темном фоне), аналогичным изображению, формируемому видеокамерой. В режиме эмуляции изображение формировалось двухкомпонентной рефракционнодифракционной оптической системой на всех длинах волн с учетом значений $M_{e . \lambda}^{(\max )}$ (табл. 2). Следует отметить, что в этом случае эмуляции, полученные без учета и с учетом влияния побочных дифракционных порядков, практически неразличимы. То есть некоторое снижение качества наблюдаемого изображения вызвано превалирующим влиянием хроматизма положения, обусловленного шириной диапазона использованного теплового излучения.

Цветовая гамма на рис. 9, $a$ отражает распределение температур по изображению нагревательного элемента (с ростом температуры цвет изменяется от синего к желтому и белому). Этот рисунок также подтверждает тот факт, что влияние побочных дифракционных порядков практически незаметно на фоне других превалирующих факторов, первым в ряду которых является хроматизм положения.

\section{Заключение}

Путем компьютерного моделирования исследовано влияние побочных дифракционных порядков микроструктуры ГГАЛ Hybrid Ge Aspheric Lens No 68-260 на качество изображения, формируемого в ИК излучении, равномерный спектральный диапазон которого ограничен длинами волн от 3 до $5 \mu \mathrm{m}$. При этом апертура ГГАЛ выбиралась таким образом, чтобы при работе ее микроструктуры в первом порядке дифракции обеспечивалось высокое качество полихроматического изображения вблизи оптической оси.

Показано, что побочные порядки дифракции ощутимо влияют на ЧКХ для бесконечно удаленной точки на оси только на низких пространственных частотах, но приводят к образованию пьедестала в дифракционном изображении, что может быть причиной образования гало. Однако если ДЭ дифракционной микроструктуры не опускается ниже 0.67 , то побочные порядки не приведут к ощутимому снижению качества изображения.

Данный вывод подтвержден и для рефракционнодифракционной двухкомпонентной оптической системы с ГГАЛ в качестве фронтального компонента и с встроенной германиевой линзой ИК видеокамеры HTPA80x64d в качестве второго компонента. Хроматизм положения и сферохроматизм этой двухкомпонентной оптической системы примерно в 3 и 6 раз превосходят соответствующие аберрации одиночной ГГАЛ, достигая уровня, типичного для ИК оптики среднего ценового диапазона. Последнее позволяет говорить об общности полученных результатов.

Компьютерное моделирование и экспериментальное исследование рефракционно-дифракционной двухкомпонентной оптической системы проводились также и при ее работе с тепловым излучением, подобным излучению абсолютно черного тела. Цветовая температура излучателя выбиралась так, что максимум его спектральной плотности энергетической светимости приходился на $\bar{\lambda}=4 \mu \mathrm{m}$. При этом на границах учитываемого при компьютерных расчетах спектрального диапазона $2 \leq \lambda \leq 8 \mu \mathrm{m}$ нормированная спектральная плотность энергетической светимости источника и ДЭ микроструктуры лежали в интервалах $0.22-0.40$ и $0.0004-0.37$ соответственно. Показано, что и в этом случае негативное влияние побочных дифракционных порядков попрежнему практически незаметно на фоне других превалирующих факторов, первым в ряду которых является хроматизм положения.

\section{Финансирование работы}

Исследование выполнено за счет гранта Российского научного фонда (проект № 20-19-00081).

\section{Конфликт интересов}

Авторы заявляет, что у него нет конфликта интересов. 


\section{Список литературы}

[1] Hu Wang, Yu Bai, Jianjun Luo. // Proc. SPIE. 2021. V. 8416. $84162 \mathrm{~N}-1$.

[2] Бездидько С.Н., Тарасишин А.В. [Электронный ресурс] Режим доступа: https://patentdb.ru/patent/2621366

[3] Грейсух Г.И., Антонов А.И., Ежсов Е.Г. // Опт. журн. 2019. T. 86. № 6. C. 3; Greisukh G.I., Antonov A.I., Ezhov E.G. // J. Optical Technology. 2019. V. 86. N 6. P. 327.

[4] Грейсух Г.И., Данилов В.А., Ежсов Е.Г., Антонов А.И., Усиевич Б.A. // Фотоника. 2020. В. 2; Greisukh G.I., Danilov V.A., Ezhov E.G., Antonov A.I., Usievich B.A. // Photonics Russia. 2020. N 2. doi 10.22184/1993-7296.FRos.2020.14.2.160.169

[5] Грейсух Г.И., Безус Е.А., Быков Д.А., Ежов Е.Г., Степанов С.A. // Опт. и спектр. 2009. Т. 106. № 4. C. 694; Greisukh G.I., Bezus E.A., Bykov D.A., Ezhov E.G., Stepanov S.A. // Opt. Spectrosc. 2009. V. 106. N 4. P. 621.

[6] Грейсух Г.И., Ежов Е.Г., Казин С.В., Сидякина З.А., Степанов С.A. // Компьютерная оптика. 2014. Т. 38. № 3. C. 418.

[7] Greisukh G.I., Danilov V.A., Ezhov E.G., Levin I.A., Stepanov S.A., Usievich B.A. // Opt. Commun. 2015. V. 338. P. 54.

[8] Грейсух Г.И., Данилов В.А., Ежов Е.Г., Степанов С.А., Усиевич Б.А. // Опт. и спектр. 2015. Т. 118. № 6. С. 997; Greisukh G.I., Danilov V.A., Ezhov E.G., Stepanov S.A., Usievich B.A. // Opt. Spectrosc. 2015. V. 118. N 6. P. 964.

[9] Грейсух Г.И., Данилов В.А., Ежсов Е.Г., Степанов С.А. // Опт. журн. 2016. Т. 83. № 3. C. 27; Greisukh G.I., Ezhov E.G., Kazin S.V., Stepanov S.A. // J. Optical Technology. 2016. V. 83. N 3. P. 163.

[10] Электронный ресурс. Режим доступа: https://www.edmundoptics.com/p/25mm-dia-x-15mm-fl-35mum-coated-hybrid-ge-aspheric-lens/22946/

[11] Коронкевич В.П., Пальчикова И.Г. // Автометрия. 1992. № 1. C. 85 .

[12] Greisukh G.I., Bobrov S.T., Stepanov S.A. Optics of diffractive and gradient-index elements and systems. Bellingham: SPIE Press, 1997. $414 \mathrm{p}$.

[13] Грейсух Г.И., Степанов С.А., Антонов А.И. // Компьютерная оптика. 2018. Т. 42. № 3. С. 369.

[14] Электронный ресурс. Режим доступа: http://www.radiantzemax.com

[15] Слюсарев Г.Г. Расчет оптических систем. Л.: Машиностроение, 1975. 639 с.

[16] Электронный ресурс. Режим доступа: https://www.heimannsensor.com/Datasheets/OverviewHTPA80x64d_Rev15.pdf

[17] Электронный ресурс. Режим доступа: https://seltokphotonics.com/upload/iblock/8f7/ 8f72e6c8cb5c8cc8cb385fa0bb0f8a29.pdf 